\title{
Steven Weinberg
}

\author{
In Memoriam
}

\author{
Sheldon Lee Glashow
}

$\mathrm{S}$

TEVEN WEINBERG and I knew each other for seventy-four of our eighty-eight years. He was my friend and classmate throughout high school and college. We met at the Bronx High School of Science, where, together with Gerald Feinberg, Morton Sternheim, and Menasha Tausner, we decided to become theoretical physicists-as we all became.

Steve and I and a few friends created the first highschool science-fiction fanzine, Etaoin Shrdlu, writing and illustrating it ourselves; but we did manage to secure a contribution from Alfred Bester, who was already admired as a writer of science fiction. Feinberg was our science editor. Our zine did not outlive our tenure at Bronx Science. To satisfy our fearsome senior class public-speaking requirement, Steve and I submitted a wire recording we made of our production of Orson Welles's 1938 radiocast, "The War of the Worlds." Steve was the narrator and I, the scientist, Dmitri Shostakovich's Seventh Symphony serving as background music.

We aced the course.

As graduation approached, Steve and I were both rejected by Harvard, but accepted by Cornell, MIT, and Princeton. To help us with this decision, Steve's father offered to drive us on a tour of the three universities. Princeton seemed too formal and MIT was too urban. We both decided to study physics at Cornell.

Steve and I thrived at Cornell. Our physics education began with a most challenging course taught by the renowned astrophysicist Kenneth Greisen. It was a course that prepared us to take advanced courses in both physics and mathematics, several at the graduate level. I lived off-campus in College Town, along with Daniel Kleitman, who later became my brother-in-law, and Kent Gordis and Daniel Filson-now both dead. Unlike Steve, we focused our studies on science and mathematics, largely ignoring the humanities. Steve was invited to live at Telluride House, an honorary fraternity. In our senior year, Steve married his college sweetheart Louise, whose birthday happens to coincide with mine. Steve chose to do his graduate study at Princeton University; I set off to study at Harvard. Julian Schwinger agreed to direct my thesis. He was convinced that the weak interactions and electromagnetism begged to be mediated by a triplet of Yang-Mills gauge bosons. "Go forth, young man, and unify!" he seemed to say. I could not meet Schwinger's challenge; but I did complete my thesis and passed my oral examination in the summer of 1958. As a National Science Foundation postdoc, I set out for a two-year stint in Copenhagen at what would become known as the Niels Bohr Institute for Theoretical Physics. There, in the spring of 1960, I met my master's challenge by identifying the algebraic structure of the electroweak synthesis and predicting the existence of novel neutral currents. The model needed four intermediaries, not just the three that Schwinger had envisaged. My model was in no way complete. Three large questions remained. How did the weak interaction intermediaries acquire their mass? Could the model describe nuclear particles as well as leptons? Was the theory renormalizable and hence mathematically consistent?

At that point, neither I nor anyone else could take my model seriously.

After a brief visit to the Bohr Institute, Steve completed his studies at Princeton, and then did a postdoc at Columbia University, and another at Berkeley, where he received a faculty position in 1960. I was just beginning my third postdoctoral year at CalTech. A few years later, both of us found ourselves as professors at Berkeley, but in 1966, we were each lured eastward: Steve to MIT, me to Harvard. We collaborated on three minor papers in the $1960 \mathrm{~s}$ prior to the publication of Steve's masterly "Model of Leptons" in 1967. This paper, with over 18,000 citations, puts to excellent use the 1964 work of Peter Higgs and several others who first investigated the spontaneous symmetry breaking of gauge theories. It was Higgs and company who discovered the mechanism by which initially massless gauge bosons acquire mass. ${ }^{1}$ Steve brilliantly inserted this mechanism into my electroweak model, the Higgs boson serving to produce the masses of the three weak intermediaries and the charged leptons.

He had elegantly answered the first of my questions.

Quarks would soon be acknowledged as the constituents of hadrons: that is to say, the nucleons and other 
strongly interacting particles. Late in 1969, John Iliopoulos, Luciano Maiani, and I, working together at Harvard, discovered that the existence of a previously conjectured fourth, charmed quark could enable the extension of the electroweak model so that it encompassed all of the known elementary particles.

Our construction answered the second of the three questions and would become known as the GIM (Glashow-Iliopoulos-Maiani) mechanism.

Gerard 't Hooft answered the third question in 1971. At this point the electroweak model, then called the Weinberg-Salam model, became a plausible theory. Many of its experimental implications were soon confirmed: Neutral currents were first detected in 1973, and evidence for the existence of the obligatory charmed quark first appeared in 1974.

Steve and I collaborated on our last and most significant paper in 1977.

I was invited to speak about the weak interactions at a conference in Stockholm in the spring of $1979 .{ }^{2}$ While in Stockholm, a member of the Nobel Physics Committee delighted in telling me that the Weinberg angle appearing in the Weinberg-Salam model was identical to the angle I introduced in my 1960 paper. I was delighted as well. Later that year, Weinberg, Abdus Salam, and I shared the 1979 Nobel Prize in Physics. Five years later, we three and our wives were invited back to Stockholm to celebrate the Nobel Prizes awarded to Carlo Rubbia and Simon van der Meer for their contributions to the observation of the weak interaction intermediaries, further confirming our electroweak theory.
Steve and I spoke only rarely since then. I regret that our long friendship and fruitful collaboration could not continue. Yet our lives had been so closely entwined that his death is not only a great loss for science, but also for me.

Sheldon Lee Glashow is a Nobel Laureate, Higgins Professor of Physics, emeritus, at Harvard University, and University Professor, emeritus, at Boston University.

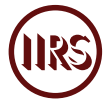

1. The others are Robert Brout, who shared the Nobel Prize with Higgs, his collaborator François Englert, and Gerald Guralnik, Richard Hagen, and Tom Kibble. Their three papers appeared in the same volume of the Physical Review, thereby leaving some uncertainty as to who among them should be credited with the discovery. Steve resolved the issue by coining the phrases "Higgs mechanism" and "Higgs boson."

2. Sheldon Lee Glashow, "Scenarios for Physics at LEP," Physics Scripta 20, no. 2 (1979): 283-88, doi:10.1088/0031$8949 / 20 / 2 / 022$. See also the prior review by Steven Weinberg, "Weak Interactions," in Proceedings, 19th International Conference on High Energy Physics 1978, Tokyo, Japan, August 23-30, 1978 (Tokyo: Physical Society of Japan, 1978), 907-18.

DOI: $10.37282 / 991819.21 .37$ 